\title{
A randomized, open label trial of methadone continuation versus forced withdrawal in a combined US prison and jail: Findings at 12 months post- release
}

\author{
Lauren Brinkley-Rubinstein ${ }^{\mathrm{a}, *}$, Michelle McKenzie ${ }^{\mathrm{b}}$, Alexandria Macmadu ${ }^{\mathrm{b}}$, Sarah Larney ${ }^{\mathrm{c}}$, \\ Nickolas Zaller ${ }^{\mathrm{d}}$, Emily Dauria ${ }^{\mathrm{e}}$, Josiah Rich ${ }^{\mathrm{f}}$

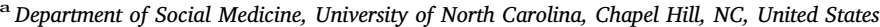 \\ b Center for Prisoner Health and Human Rights, The Miriam Hospital, Providence, RI, United States \\ ${ }^{\mathrm{c}}$ National Drug and Alcohol Research Centre, University of New South Wales, Sydney, Australia \\ d College of Public Health, University of Arkansas for Medical Sciences, Little Rock, AR, United States \\ e Department of Psychiatry, University of California, San Francisco, CA, United States \\ ${ }^{\mathrm{f}}$ Division of Infectious Diseases, The Miriam Hospital, Providence, RI, United States
}

\section{A R T I C L E I N F O}

Keywords:

Methadone maintenance treatment

Incarceration

\begin{abstract}
A B S T R A C T
Recently incarcerated individuals are at increased risk of opioid overdose. Methadone maintenance treatment (MMT) is an effective way to address opioid use disorder and prevent overdose; however, few jails and prisons in the United States initiate or continue people who are incarcerated on MMT. In the current study, the 12 month outcomes of a randomized control trial in which individuals were provided MMT while incarcerated at the Rhode Island Department of Corrections (RIDOC) are assessed. An as-treated analysis included a total of 179 participants -128 who were, and 51 who were not, dosed with methadone the day before they were released from the RIDOC. The results of this study demonstrate that 12 months post-release individuals who received continued access to MMT while incarcerated were less likely to report using heroin and engaging in injection drug use in the past 30 days. In addition, they reported fewer non-fatal overdoses and were more likely to be continuously engaged in treatment in the 12-month follow-up period compared to individuals who were not receiving methadone immediately prior to release. These findings indicate that providing incarcerated individuals continued access to MMT has a sustained, long-term impact on many opioid-related outcomes postrelease.
\end{abstract}

\section{Introduction}

Prevalence of opioid use disorder (OUD) is exaggerated among those who are incarcerated (Mumola and Karberg, 2006). Just over $23 \%$ of state prisoners report ever using heroin or other opiates and $13 \%$ report regular use prior to incarceration (Mumola and Karberg, 2006). In addition, people who have recently been incarcerated are at extreme risk of overdose during community re-entry (Binswanger et al., 2007). A recent study that investigated all causes of mortality of people who were formerly incarcerated in Washington State found that overdose was the number one cause of death (Binswanger et al., 2013).

Methadone-maintenance treatment (MMT), the combination of behavioral therapy, counseling and methadone provision, is an effective, evidence-based approach to address opioid use disorder and overdose
(Connock et al., 2007). Numerous studies have documented the farreaching benefits to implementing MMT in correctional populations, including post-incarceration reductions in illicit opioid use (Mattick et al., 2009; Kinlock et al., 2009), re-incarceration (Deck et al., 2009; Larney et al., 2012), mortality and overdose (Degenhardt et al., 2011; Kerr et al., 2007), and HIV risk behaviors (MacArthur et al., 2012) among others (Rich et al., 2015, Zaller et al., 2013, McKenzie et al., 2012, Heimer et al., 2006; Dolan et al., 2003).

In the United States (US), there are over 3200 local and county jails and 1800 state and federal prisons, but few facilities offer addiction treatment using MMT (Vestal, 2016; Lee et al., 2015). In 2008, less than $0.1 \%$ of the total prison population received any form of buprenorphine or MMT (Larney and Dolan, 2009), and, while 28 state prison systems make MMT available to those who are incarcerated, a majority restrict

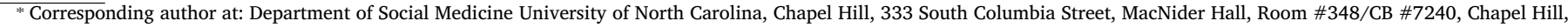
NC 27599-7240, United States.

E-mail address: Lauren_Brinkley@med.unc.edu (L. Brinkley-Rubinstein).
} 
treatment to special populations (e.g., pregnant women; Nunn et al., 2009).

When MMT or other forms of medication for addiction treatment (MAT) (Wakeman, 2017; e.g., buprenorphine) are not provided in the correctional setting, individuals who are addicted to opioids experience symptoms of withdrawal. Opioid withdrawal can include severe physical discomfort and psychological distress, risk of suicide, and leads to loss of opioid tolerance, thereby increasing risk of fatal and non-fatal overdose post-release (Merrell et al., 2010). Also, while the current literature points to the clear benefit of providing MMT during incarceration and linkage to treatment in the community, less is known about the long-term effects of MMT access during incarceration.

The objective of the current study was to identify the long-term effects of providing access to MMT for people who are incarcerated. From 2011-2014, we conducted a randomized control trial to assess the impact of continued MMT versus forced withdrawal from methadone in people who were incarcerated for six months or less, on fatal and nonfatal overdose, substance use, emergency department use, treatment engagement in the community, and HIV risk behaviors such as injection drug use and transactional sex. Baseline results indicated that forced withdrawal from MMT reduced the likelihood of MMT engagement post-release in the community (Rich et al., 2015). In the current study, we present outcomes measured at 12 months following release.

\section{Methods}

\subsection{Study design}

The study was conducted at the Rhode Island Department of Corrections (RIDOC), a unified, statewide prison and jail system. All participants gave written informed consent. This study was approved by the Institutional Review Board of the Miriam Hospital in Providence, Rhode Island (RI), and the RIDOC Medical Research Advisory Group. In addition, the study was reviewed by a data safety monitoring board every six months for the first two years of recruitment, then once per year until the study ended. This trial is registered with ClinicalTrials.gov (number NCT01874964).

Inclusion criteria for the study included being incarcerated for at least one week and no more than six months and having been engaged in MMT treatment prior to incarceration. At intake, individuals reported to RIDOC nursing staff whether they were enrolled in a MMT program before incarceration. Nursing staff then confirmed dosing with the community provider. Nursing staff and MMT counselors provided study information to eligible participants. The name and facility location of individuals who expressed interest were forwarded to the research staff who then followed up with an in-person visit that occurred within seven days.

\subsection{Enrollment and randomization}

After consent was obtained, participants were randomly assigned (1:1) using a computer-generated random permutation to either a) continued MMT or b) tapered withdrawal from methadone after the first week of incarceration, the standard of care at the RIDOC at the time. Study staff worked in tandem and randomly assigned participants to each group-meaning that separate study staff members completed enrollment and randomization. The same staff member who enrolled the participant followed up with him or her in the community postrelease. Because there were more men than women and few racial minorities were enrolled in MMT and incarcerated during the study period, urn randomization was used to stratify individuals on the basis of sex and race. Urn randomization is appropriate, given that it can balance groups with several covariates and has a low risk of experimenter bias or manipulation (Wei and Lachin, 1988).

\subsection{Procedures}

Participants in the MMT continuation group were maintained on methadone during incarceration, with adjustments made to their dose as clinically indicated. Participants who were receiving a stable dose were continued on the same dose. Those participants whose doses were being adjusted at the time of incarceration or who had symptoms caused by doses that were either too low or too high, had adjustments made in accordance with standard clinical practices, usually in collaboration with their community methadone clinic.

Participants who were assigned to standard care (meaning forced withdrawal from methadone) completed the RIDOC's standard protocol for MMT upon entry, which, at the time of the study, included continuation of entry dose during the first week of incarceration followed by a tapered withdrawal schedule (e.g., an entry dose of $>100 \mathrm{mg}$ would be reduced by $5 \mathrm{mg}$ per day to $100 \mathrm{mg}$, then reduced by $3 \mathrm{mg}$ per day to $0 \mathrm{mg}$ ). Since the standard taper protocol typically lasted 4-6 weeks or longer, participants in the forced withdrawal group could still be receiving a daily dose of methadone at the time of release, dependent on the length of their incarceration and starting dose (e.g., if they were incarcerated for two weeks). Upon discharge (regardless of study condition), all participants were assisted with transportation, scheduling, and financing for their first MMT appointment in the community.

All research assessments were administered via face-to-face interviews. At enrollment, we asked participants to provide multiple ways to contact them in the community to increase our chances to conduct follow-up interviews. Participants provided thorough contact information and information about places they liked to "hangout". This information was used if phone and mail attempts went unanswered and was particularly useful for unstably housed participants. To accommodate transportation issues, we provided cab or bus fare and met participants in locations convenient for them to complete the interview. In addition, we provided participants with business cards with interview dates, reimbursement amount, and research staff contact information and reminded them of assessments via mail and phone. Research assessments were conducted at one, six, and 12 months postrelease. All outcomes reported herein are from the 12-month follow-up interview.

All follow-up interviews took place at a location most convenient to the participant, such as a private interview space located at The Miriam Hospital or one of the treatment facilities, a fast food restaurant, or a participant's home. Privacy and safety concerns were part of staff training and were discussed in staff meetings. Follow-up interviews, on average, lasted about thirty minutes. After each completed assessment, participants received $\$ 20$. Additionally, participants could receive $\$ 5$ for checking in between the one month and six-month study visit and between the six and 12-month study visit.

\subsection{Study population}

We enrolled participants between June 2011 and April 2013. A detailed description of the study population and one-month outcomes are published elsewhere (Rich et al., 2015). At baseline, there were a total of 223 participants, and 179 completed a 12-month follow-up ( $80.3 \%$ retention). Of the 44 participants for whom no 12-month follow-up interviews were completed, four died of an overdose (two were on MMT at release and two were not) and one participant died from violent causes. Seven participants were continuously incarcerated between the six- and 12- month assessments, and did not complete a 12month interview. One participant refused participation in the 12-month assessment, and we were unable to contact the remaining 31 participants. There were no statistically significant differences between those who completed a 12-month interview and those who were lost to follow-up (data not shown). A total of 51 participants were released from incarceration after having been completely tapered from methadone. These participants, on average, spent 52 days without methadone 


\begin{tabular}{|c|c|c|c|c|}
\hline & Total $(\mathrm{N}=179)$ & $\begin{array}{l}\text { Dosed with methadone day before release } \\
n=128\end{array}$ & $\begin{array}{l}\text { Not dosed with methadone day before release } \\
n=51\end{array}$ & $p$-value \\
\hline \multicolumn{5}{|l|}{ Sex } \\
\hline Male & $140(78.2 \%)$ & $98(76.6 \%)$ & $42(82.4 \%)$ & 0.3986 \\
\hline Female & $39(21.8 \%)$ & $30(23.4 \%)$ & $9(17.7 \%)$ & 0.3986 \\
\hline \multicolumn{5}{|l|}{ Ethnic origin } \\
\hline White & $141(78.8 \%)$ & $101(78.9 \%)$ & $40(78.4 \%)$ & 0.9439 \\
\hline Black & $8(4.5 \%)$ & $5(3.9 \%)$ & $3(5.9 \%)$ & 0.5661 \\
\hline Other & $30(16.8 \%)$ & $22(17.2 \%)$ & $8(15.7 \%)$ & 0.5501 \\
\hline Hispanic & $25(14.0 \%)$ & $19(14.8 \%)$ & $6(11.8 \%)$ & 0.5925 \\
\hline Age at baseline (years), median (IQR) & $32.6(28.4,40.9)$ & $32.1(27.8,40.6)$ & $35.4(30.3,42.2)$ & 0.0843 \\
\hline $\begin{array}{l}\text { Duration of incarceration (days), median } \\
\text { (IQR) }\end{array}$ & $45(16,79)$ & $30.0(14.0,58.5)$ & $80(57.0,116.0)$ & $<0.001$ \\
\hline \multicolumn{5}{|l|}{ Number of years in education } \\
\hline Did not finish high school & $71(39.7 \%)$ & $52(40.6 \%)$ & $19(37.3 \%)$ & 0.6775 \\
\hline Finished high school & $68(38.0 \%)$ & $48(37.5 \%)$ & $20(39.2 \%)$ & 0.8310 \\
\hline College or higher education & $40(22.4 \%)$ & $28(21.9 \%)$ & $12(23.5 \%)$ & 0.8105 \\
\hline
\end{tabular}

while incarcerated. The range was from 1 to 130 days without methadone.

\subsection{Measures}

\subsubsection{Socio-demographics}

Self-reported sociodemographic data collected included sex, age, race/ethnicity, health insurance status, and educational attainment

\subsubsection{Drug treatment}

At enrollment, all participants provided written consent for the research team to access their methadone records at community clinics post-release. We used these data to assess treatment engagement and retention throughout the study period. We also measured self-reported engagement in other treatment modalities (e.g., outpatient and residential) and engagement in peer support programs such as Alcoholics Anonymous or Narcotics Anonymous. Continuous enrollment in MMT was defined as being in treatment for at least 335 days during the 12month study period (allowing for 30 days post-release to enroll in treatment) based on MMT administrative records (matched using first and last name and date of birth).

\subsubsection{Substance use behaviors, overdose, and HIV risk}

Substance use data were obtained via the Addiction Severity Index (ASI). The ASI is a self-report questionnaire that asks about drug use in the past 30 days (McLellan et al., 1980). HIV risk behaviors (engagement in transactional sex and injection drug use in the past 30 days) were also measured via self-report. Similarly, non-fatal overdose was assessed via self-report, but participants were asked to report any nonfatal overdoses during the entire (12 month) follow-up period. Fatal overdose was determined through medical examiner reports (matched using first and last name and date of birth).

\subsubsection{Legal status}

We asked participants to self-report re-arrest and re-incarceration. In addition, we obtained re-incarceration data from RIDOC (matched using first and last name and date of birth), which are publically available upon request.

\subsubsection{Emergency department usage}

Participants self-reported utilizing the emergency department in the past 12 months. Additionally, participants signed releases, permitting the study team to obtain emergency department data (matched using first and last name and date of birth) from RI's largest hospital facilities.

\subsection{Outcomes and analysis}

For this analysis, outcomes of interest at 12 months included engagement in MMT, re-arrest, re-incarceration, fatal and non-fatal overdose, emergency department usage, substance use, and HIV risk behaviors (including transactional sex and injection drug use). We analyzed outcomes with the $\chi^{2}$ test to assess differences between those who were 1) randomized to MMT or forced withdrawal from methadone (intent-to-treat) and 2) on MMT at release and those who were not (as-treated).

The intent-to-treat analysis included all eligible participants in the study as randomized. The as-treated analysis included all eligible participants in the study by their methadone status on the day before their release: either receiving any dose of methadone or not receiving methadone. This type of analysis was conducted because participants in the forced withdrawal group could still be receiving some amount of methadone just before their release if they had not yet completed the RIDOC's withdrawal protocol (see Rich et al., 2015 for a more in-depth explanation of randomization and baseline procedures).

\section{Results}

A majority of participants who completed the 12-month follow-up were male $(78.2 \%)$, White $(78.8 \%)$, and had not finished high school (39.7\%). The median age of all participants at baseline was 32.6 years [IQR: 28.4, 40.9]. See Table 1 for all participant demographic details. Those who received methadone on the day before release were significantly more likely than those not dosed with methadone on the day before release to have been incarcerated for a shorter period of time. When analyzed by randomization status (MMT versus forced withdrawal), no significant differences were found between groups (data not shown).

\subsection{Intent-to-treat analysis}

Of the participants assigned to the continued MMT group ( $\mathrm{n}=96)$, $43.6 \%$ were engaged in continuous MMT at 12 months, whereas only $38.8 \%$ of the forced withdrawal group $(n=83)$ were. A lower proportion of those in the continued MMT group were re-incarcerated, utilized the emergency department, used heroin, and reported injection drug use at 12 months than those in the forced withdrawal group; however, none of these differences were significant (see Table 2 for all intent-to-treat analyses). 


\begin{tabular}{|c|c|c|c|}
\hline & Continued methadone $\mathrm{n}=96$ & $\begin{array}{l}\text { Forced methadone withdrawal } \\
\mathrm{n}=83\end{array}$ & Unadjusted Odds Ratio (OR) $(95 \% \mathrm{CI})$ \\
\hline \multicolumn{4}{|l|}{ Drug treatment (prior 12 months) } \\
\hline Outpatient drug-free program & $21(21.9 \%)$ & $12(14.5 \%)$ & $1.66(0.76,3.61)$ \\
\hline Residential treatment program & $23(24.0 \%)$ & $12(14.5 \%)$ & $1.86(0.86,4.03)$ \\
\hline Prison/jail treatment program & $1(1.0 \%)$ & $1(1.2 \%)$ & $0.86(0.05,14.01)$ \\
\hline Self-help/12-step program & $37(38.5 \%)$ & $31(37.4 \%)$ & $1.05(0.57,1.93)$ \\
\hline In methadone treatment program & $87(90.6 \%)$ & $73(88.0 \%)$ & $1.32(0.51,3.43)$ \\
\hline In any drug treatment program & $90(93.8 \%)$ & $76(91.6 \%)$ & $1.38(0.44,4.29)$ \\
\hline Detox program (prior 12 months) & $16(16.7 \%)$ & $13(15.7 \%)$ & $1.08(0.48,2.39)$ \\
\hline $\begin{array}{l}\text { Continuous engagement in methadone treatment program } \\
(\mathrm{n}=174)\end{array}$ & $41(43.6 \%)$ & $31(38.8 \%)$ & $1.22(0.67,2.24)$ \\
\hline Health insurance (prior 12 months) & $46(47.9 \%)$ & $49(59.0 \%)$ & $0.64(0.35,1.16)$ \\
\hline \multicolumn{4}{|l|}{ Opioid replacement therapy (prior 30 days) } \\
\hline Prescribed methadone & $64(66.7 \%)$ & $56(67.5 \%)$ & $0.96(0.52,1.80)$ \\
\hline Prescribed buprenorphine & $2(2.1 \%)$ & $6(7.2 \%)$ & $0.27(0.05,1.39)$ \\
\hline \multicolumn{4}{|l|}{ Adverse events (prior 12 months) } \\
\hline Re-arrested & $47(49.0 \%)$ & $47(56.6 \%)$ & $0.74(0.41,1.33)$ \\
\hline Reincarcerated (self-report) & $26(27.1 \%)$ & $31(37.4 \%)$ & $0.62(0.33,1.17)$ \\
\hline Reincarcerated (administrative data) & $55(57.3 \%)$ & $48(57.8 \%)$ & $0.98(0.54,1.77)$ \\
\hline Emergency department utilization (self-report) & $41(42.7 \%)$ & $37(44.6 \%)$ & $0.93(0.51,1.68)$ \\
\hline Emergency department utilization(administrative data) & $36(37.5 \%)$ & $34(41.0 \%)$ & $0.87(0.47,1.58)$ \\
\hline \multicolumn{4}{|l|}{ Drug use at 12 months (prior 30 days) } \\
\hline Heroin & $27(28.1 \%)$ & $24(28.9 \%)$ & $0.96(0.50,1.84)$ \\
\hline Prescription opioids & $12(12.5 \%)$ & $12(14.5 \%)$ & $0.85(0.36,2.00)$ \\
\hline Alcohol to feel effects & $21(21.9 \%)$ & $13(15.7 \%)$ & $1.51(0.70,3.24)$ \\
\hline Cannabis use & $30(31.3 \%)$ & $26(31.3 \%)$ & $1.00(0.53,1.88)$ \\
\hline Other drug use $\mathrm{b}^{\mathrm{b}}$ & $36(37.5 \%)$ & $42(50.6 \%)$ & $0.59(0.32,1.06)$ \\
\hline \multicolumn{4}{|l|}{ HIV risk behaviors (prior 30 days) } \\
\hline Injection drug use & $20(20.8 \%)$ & $23(27.7 \%)$ & $0.69(0.35,1.37)$ \\
\hline Transactional sex ${ }^{c}$ & $2(2.1 \%)$ & $1(1.2 \%)$ & $1.75(0.16,19.59)$ \\
\hline Overdoses (nonfatal) & $6(6.3 \%)$ & $12(14.5 \%)$ & $0.39(0.14,1.10)$ \\
\hline
\end{tabular}

$* p<.05$.

$\mathrm{a}=$ Defined as $\geq 335$ total days in treatment during study period; 5 records were excluded due to incomplete data.

$\mathrm{b}$ = Includes use of barbiturates; sedatives, hypnotics, or tranquilizers; benzodiazepines; cocaine or crack; amphetamines; hallucinogens; or inhalants.

$\mathrm{c}=$ Includes sex in exchange for drugs, money, or food/shelter.

Abbreviations;: IQR = interquartile range; $\mathrm{CI}=$ confidence interval.

\subsection{As-treated analysis}

When data were analyzed by methadone status at release (receiving or not receiving methadone), at 12 month follow-up, those who received MMT on the day before release $(n=128)$ were significantly less likely than those not dosed with methadone the day before release $(\mathrm{n}=51)$ to report using heroin $(p=.0467)$ and injecting drugs in the past 30 days $(p=.0033)$, and were significantly less likely to have had a non-fatal overdose in the 12 months follow-up period (7\% vs $18 \%$, $p=.0390$ ). Those who received methadone on the day before release were significantly more likely than those not dosed with methadone on the day before release to engage continuously in MMT during the 12month follow-up period $(p=.0211)$ (see Table 3$)$. There were no differences between study groups for re-incarceration, visits to the emergency department, transactional sex, or use of prescription opioids, alcohol, cannabis, or other drugs.

\section{Discussion}

The objective of this study was to identify the long-term (12 months) effects of providing access to MMT to people who are incarcerated before their release into the community. The results of this study demonstrate that 12 months post-release individuals who received MMT while incarcerated were less likely to report heroin and injection drug use in the past 30 days and experienced fewer non-fatal overdoses. They were also more likely to be continuously engaged in MMT in the community. These findings indicate that providing incarcerated individuals access to MMT prior to release with linkage to treatment in the community is a successful strategy to address the growing opioid epidemic and lessen long-term risk.
Our results corroborate previous research from our group and others that demonstrate the benefits of MMT among those with recent incarceration (Rich et al., 2015, Kinlock et al., 2008). Access to opioid pharmacotherapies while incarcerated significantly reduces mortality, both while incarcerated and post-release, and decreases likelihood of engaging in HIV risk behaviors (Marsden et al., 2017; McKenzie et al., 2012; Degenhardt et al., 2014; Larney et al., 2014; Larney, 2010). In addition, incarceration is often cited as a factor that can increase risk behavior. For instance, those with a history of incarceration are more likely to share used needles during community reentry (Milloy et al., 2009) and individuals leaving incarceration have a multi-fold increased risk of overdose than the general population and at other times of active drug use (Binswanger et al., 2013; Connock et al., 2007; Wakeman, 2017). Therefore, providing MMT and other forms of MAT may decrease mortality and risk behaviors and negate the possible adverse effect incarceration itself can have on substance use related health outcomes. However, to date, robust MMT programs in correctional settings are rare in the US. This study contributes to a growing body of evidence by demonstrating that access to MMT during incarceration can have a sustained long-term impact.

While we did find that those who received MMT on the day before their release were more likely to engage in continuous treatment during the follow- up period, the effects were modest. Only $45 \%$ of those who received MMT the day before release and $26 \%$ of those who did not were continuously engaged in treatment. Financial stressors may have impacted access to treatment in the community long-term. At the time of the study, most individuals leaving incarceration did not have medical insurance, as many were in enrolled in a state funded treatment program, to which they lost access while incarcerated. To ensure that financial concerns were not a barrier immediately upon release, the 


\begin{tabular}{|c|c|c|c|}
\hline & $\begin{array}{l}\text { Dosed with methadone day before } \\
\text { release } n=128\end{array}$ & $\begin{array}{l}\text { Not dosed with methadone day before } \\
\text { release } n=51\end{array}$ & $\begin{array}{l}\text { Unadjusted Odds Ratio (OR) } \\
\text { (95\% CI) }\end{array}$ \\
\hline \multicolumn{4}{|l|}{ Drug treatment (prior 12 months) } \\
\hline Outpatient drug-free program & $28(21.9 \%)$ & $5(9.8 \%)$ & $0.39(0.14,1.07)$ \\
\hline Residential treatment program & $24(18.8 \%)$ & $11(21.6 \%)$ & $1.19(0.53,2.65)$ \\
\hline Prison/jail treatment program & $0(0 \%)$ & $2(3.9 \%)$ & - \\
\hline Self-help/12-step program & $46(35.9 \%)$ & $22(43.1 \%)$ & $1.35(0.70,2.62)$ \\
\hline In methadone treatment program & $117(91.4 \%)$ & $43(84.3 \%)$ & $0.51(0.19,1.34)$ \\
\hline In any drug treatment program & $120(93.8 \%)$ & $46(90.2 \%)$ & $0.61(0.19,1.97)$ \\
\hline Detox program (prior 12 months) & $21(16.4 \%)$ & $8(15.7 \%)$ & $0.95(0.39,2.30)$ \\
\hline $\begin{array}{l}\text { Continuous engagement in methadone treatment } \\
\operatorname{program}^{\mathrm{a}}(\mathrm{n}=174)\end{array}$ & $56(45.2 \%)$ & $13(26.0 \%)$ & $0.43(0.21,0.88)^{*}$ \\
\hline Health insurance (prior 12 months) & $67(52.3 \%)$ & $28(54.9 \%)$ & $1.11(0.58,2.13)$ \\
\hline \multicolumn{4}{|l|}{ Opioid replacement therapy (prior 30 days) } \\
\hline Prescribed methadone & $88(68.8 \%)$ & $32(62.8 \%)$ & $0.77(0.39,1.51)$ \\
\hline Prescribed buprenorphine & $4(3.1 \%)$ & $4(7.8 \%)$ & $2.64(0.63,11.0)$ \\
\hline \multicolumn{4}{|l|}{ Adverse events (prior 12 months) } \\
\hline Re-arrested & $65(50.8 \%)$ & $29(56.9 \%)$ & $1.28(0.67,2.46)$ \\
\hline Reincarcerated (self-report) & $39(30.5 \%)$ & $18(35.3 \%)$ & $1.25(0.63,2.47)$ \\
\hline Reincarcerated (administrative data) & $72(56.3 \%)$ & $31(60.8 \%)$ & $1.21(0.62,2.34)$ \\
\hline Emergency department utilization (self-report) & $52(40.6 \%)$ & $26(51.0 \%)$ & $1.52(0.79,2.92)$ \\
\hline $\begin{array}{l}\text { Emergency department utilization (administrative } \\
\text { data) }\end{array}$ & $52(40.6 \%)$ & $18(35.3 \%)$ & $0.80(0.41,1.56)$ \\
\hline \multicolumn{4}{|l|}{ Drug use at 12 months (prior 30 days) } \\
\hline Heroin & $31(24.2 \%)$ & $20(39.2 \%)$ & $2.02(1.01,4.04)^{*}$ \\
\hline Prescription opioids & $18(14.1 \%)$ & $6(11.8 \%)$ & $0.82(0.30,2.19)$ \\
\hline Alcohol to feel effects & $25(19.5 \%)$ & $9(17.7 \%)$ & $0.88(0.38,2.05)$ \\
\hline Cannabis use & $45(35.2 \%)$ & $11(21.6 \%)$ & $0.51(0.24,1.08)$ \\
\hline Other drug use $\mathrm{b}^{\mathrm{b}}$ & $50(39.1 \%)$ & $28(54.9 \%)$ & $1.90(0.99,3.66)$ \\
\hline \multicolumn{4}{|l|}{ HIV risk behaviors (prior 30 days) } \\
\hline Injection drug use & $23(18.0 \%)$ & $20(39.2 \%)$ & $2.95(1.43,6.06)^{*}$ \\
\hline Transactional sex ${ }^{c}$ & $2(1.6 \%)$ & $1(2.0 \%)$ & $1.26(0.11,14.2)$ \\
\hline Overdoses (nonfatal) & $9(7.0 \%)$ & $9(17.7 \%)$ & $2.83(1.05,7.61)^{*}$ \\
\hline
\end{tabular}

$* p<.05$.

$\mathrm{a}=$ Defined as $\geq 335$ total days in treatment during study period; 5 records were excluded due to incomplete data

$\mathrm{b}=$ Includes use of barbiturates; sedatives, hypnotics, or tranquilizers; benzodiazepines; cocaine or crack; amphetamines; hallucinogens; or inhalants.

$\mathrm{c}=$ Includes sex in exchange for drugs, money, or food/shelter.

Abbreviations;: IQR = interquartile range; $\mathrm{CI}=$ confidence interval.

study paid for the first 10-weeks of MMT for participants who did not have insurance; however, financial limitations could have restricted access to MMT in the community after 10 weeks. In RI, because of the passage of the Affordable Care Act and the subsequent expansion of Medicaid, many people released from the RIDOC are now eligible for health insurance, rendering costs less of an issue. However, in many states with the highest rates of incarceration, Medicaid was not expanded, and the cost of MMT continues to be a barrier to treatment engagement and sustainment in those states (Cuellar and Cheema, 2012; Rich et al., 2014). It is imperative that post-release linkage to MMT is treatment is included in future MMT programs. In addition, supportive services that address financial and other barriers that may prohibit long term engagement in MMT should be considered essential.

Many studies have demonstrated that access to MAT during incarceration can lessen short-term risk of overdose and risky opioid use behaviors (Rich et al., 2015; Zaller et al., 2013; McKenzie et al., 2012). Our results extend these findings and demonstrate that access to MMT can lessen risk in the first-year post-release and affect engagement in continuous MMT in the community. In response to the increasing number of overdose deaths in the US, many states have developed strategic initiatives to lower risk. However, few jails and prisons offer comprehensive MAT programs, and to our knowledge, RI is one of the only states that has explicitly incorporated corrections-based recommendations into their overdose reduction plan. In 2015, the governor of RI created the Overdose Prevention and Intervention Task Force with the goal of reducing opioid overdose deaths by one third after three years (Rhode Island Governor's Overdose Prevention and Intervention Task Force, 2015). A key component of the task force's recommendations was a comprehensive MAT initiative that included making available methadone, buprenorphine, and naltrexone to all eligible individuals. The findings of the current study provide evidence to support the creation and expansion of similar comprehensive programs in other correctional settings across the country.

\subsection{Study limitations and other considerations}

We could not control for the duration of incarceration, and many (41\%) participants who were assigned to the forced withdrawal group were released before completing the standard MMT taper protocol. At the time that this study was conducted, the standard taper protocol was to gradually taper methadone at RIDOC; however, in most US jurisdictions, MMT is abruptly stopped upon incarceration, which might lead to an even greater effect of MMT access for those incarcerated for shorter periods. The results of the as-treated analysis lend support to this theory.

Several factors may limit the generalizability of this study. Recruiting and conducting research in correctional facilities can present significant challenges (McKenzie et al., 2009). Because of this, our sample size was smaller than anticipated. Also, this study was conducted in RI where a larger proportion of incarcerated individuals are White, which is very different than other states (Zaller et al., 2009). In addition, this study took place at the RIDOC, a unique state-wide unified prison and jail system, and only included participants who were at the RIDOC for six months or less; consequently, prisoners with longer sentences were not included. We acknowledge that there were differences in length of sentence between those who received methadone on the day before release and those who did not. Length of sentence has been shown to play a role in risk of overdose post-release as those who 
are incarcerated longer are at higher risk of losing their opioid tolerance (Merrell et al., 2010). However, while length of incarceration was an important factor, dose amount at the time of incarceration also played a role. Individuals who began their incarceration at a lower dose would have been tapered more quickly than individuals who began their incarceration at a higher dose.

In the current study, we did not find associations between access to MMT while incarcerated and re-incarceration, emergency department usage, transactional sex, or treatment engagement post-release. Low numbers of individuals in both conditions reported engaging in transactional sex, and this may explain its non-association with MMT. In addition, structural factors (e.g., policing practices or access to healthcare), often outside of an individual's control, may have affected emergency department usage and reincarceration. While previous studies have found an association between reincarceration and medication for addiction treatment, rates of arrest and incarceration vary widely and relate heavily to local and state policies and laws (Rich et al., 2015). RI has the 4th highest rate of probation in the country (Herberman and Bonczar, 2015), therefore, many more participants may have had broader contact with the criminal justice system that was not captured in the current study.

While only 179 participants completed the 12-month follow-up, we had access to administrative data related to reincarceration, emergency department usage and treatment engagement for the entire baseline sample of 223 individuals; however, these administrative data are incomplete and do not include information about treatment, emergency department use, or reincarceration in locations that were outside the scope of the current study. In addition, if individuals used an alias or did not provide an accurate name or date of birth, then we would have been unable to identify them using only administrative data. We did, though, complete an assessment of the differences in these administrative data variables for those who completed a 12-month follow-up (179) and those who did not (44), and we found no statistically significant differences.

\section{Conclusion}

The US is experiencing an epidemic of escalating opioid use, and those who have recently been incarcerated are at increased risk of overdose and risky drug use post-release. Nonetheless, access to MMT in correctional settings or upon discharge remains rare in the US. Our findings indicate that providing MMT to individuals who are incarcerated can affect long-term outcomes including continuous treatment engagement, using heroin, injecting drugs, and non-fatal overdose in the first year after release. Based on the findings of the current study, more states should partner with correctional institutions to create or expand programs that provide incarcerated, opioid dependent individuals with access to MMT and provide linkage to treatment programs in the community upon release.

\section{Contributors}

All authors made significant contributions to the study's design and conduct. L-BR (lead writer), MM, and AM shared responsibility in developing initial drafts and writing this report. AM conducted study analyses. NZ, ED, SL, and JDR critiqued the analysis plan, assisted with the interpretation of findings, and contributed to multiple versions of this brief. All authors contributed to and have approved the final manuscript for publication.

\section{Role of funding source}

This work is supported by NIH grant R21DA029201. The work of Dr. Rich is also supported by NIH grants K24DA022112 and P30AI042853. Dr. Brinkley-Rubinstein is supported by the UCLA HIV/ AIDS, Substance Abuse and Trauma Training Program (HA-STTP)
(R25DA035692), and the Lifespan/Brown Criminal Justice Research Program on HIV and Substance Use (R23DA037190). The funders had no role in the design, analysis, interpretation, writing, review, preparation, or decision to publish this manuscript.

\section{Conflict of interest}

No conflict declared.

\section{References}

Binswanger, I.A., Stern, M.F., Deyo, R.A., Heagerty, P.J., Cheadle, A., Elmore, J.G., Koepsell, T.D., 2007. Release from prison - a high risk of death for former inmates. New Engl. J. Med. 356, 157-165.

Binswanger, I.A., Blatchford, P.J., Mueller, S.R., Stern, M.F., 2013. Mortality after prison release: opioid overdose and other causes of death, risk factors, and time trends from 1999 to 2009. Ann. Intern. Med. 159, 592-600.

Connock, M., Juarez-Garcia, A., Jowett, S., et al., 2007. Methadone and buprenorphine for the management of opioid dependence: a systematic review and economic evaluation. Health Technol. Assess. 11, 1-171.

Cuellar, A.E., Cheema, J., 2012. As roughly 700000 prisoners are released annually, about half will gain health coverage and care under federal laws. Health Aff. 31, 931-938.

Deck, D., Wiitala, W., McFarland, B., et al., 2009. Medicaid coverage, methadone maintenance, and felony arrests: outcomes of opiate treatment in two states. J. Addict. Dis. 28, 89-102.

Degenhardt, L., Bucello, C., Mathers, B., et al., 2011. Mortality among regular or dependent users of heroin and other opioids: a systematic review and meta-analysis of cohort studies. Addiction 106, 32-51.

Degenhardt, L., Larney, S., Kimber, J., et al., 2014. The impact of opioid substitution therapy on mortality post-release from prison: retrospective data linkage study. Addiction 109, 1306-1317.

Dolan, K.A., Shearer, J., MacDonald, M., Mattick, R.P., Hall, W., Wodak, A.D., 2003. A randomised controlled trial of methadone maintenance treatment versus wait list control in an Australian prison system. Drug Alcohol Depend. 72, 59-65.

Heimer, R., Catania, H., Newman, R. G., Zambrano, J., Brunet, A., Ortiz, A.M., 2006. Methadone maintenance in prison: evaluation of a pilot program in Puerto Rico. Drug Alcohol Depend. 83, 122-129.

Herberman, E.J., Bonczar, T.P., 2015. Probation and Parole in the United States, 2013. Bureau of Justice Statistics, Washington DC.

Kerr, T., Fairbairn, N., Tyndall, M., et al., 2007. Predictors of non-fatal overdose among a cohort of polysubstance-using injection drug users. Drug Alcohol Depend. 87, 39-45.

Kinlock, T.W., Gordon, M.S., Schwartz, R.P., O'Grady, K.E., 2008. A study of methadone maintenance for male prisoners: 3-month postrelease outcomes. Crim. Justice Behav. 35, 34-47. http://dx.doi.org/10.1177/0093854807309111.

Kinlock, T.W., Gordon, M.S., Schwartz, R.P., Fitzgerald, T.T., O'Grady, K.E., 2009. A randomized clinical trial of methadone maintenance for prisoners: results at 12 months postrelease. J. Subst. Abuse Treat. 37, 277-285.

Larney, S., Dolan, K., 2009. A literature review of international implementation of opioid substitution treatment in prisons: equivalence of care? Eur. Addict. Res. 15 (107), 20.

Larney, S., et al., 2012. Effect of prison-based opioid substitution treatment, and postrelease retention in treatment, on risk of re-incarceration. Addiction 107, 372-380.

Larney, S., Gisev, N., Farrell, M., et al., 2014. Opioid substitution therapy as a strategy to reduce deaths in prison: retrospective cohort study. BMJ Open 4, e004666.

Larney, S., 2010. Does opioid substitution treatment in prison reduce injecting-related HIV risk behaviours. A systematic review. Addiction 105, 216-223.

Lee, J.D., McDonald, R., Grossman, E., et al., 2015. Opioid treatment at release from jail using extended-release naltrexone: a pilot proof-of-concept randomized effectiveness trial. Addiction 110, 1008-1014.

MacArthur, G.J., Minozzi, S., Martin, N., et al., 2012. Opiate substitution treatment and HIV transmission in people who inject drugs: systematic review and meta-analysis. BMJ 45, e5945.

Marsden, J., Stillwell, G., Hayley, Jones, Cooper, A., Eastwod, B., Farrell, M., Lowden, T., Maddalena, N., Metcalfe, C., Shaw, J., 2017. Does exposure to opioid substitution treatment in prison reduce the risk of death after release? A national prospective observational study in England. Addiction 112, 1408-1418.

Mattick, R.P., Breen, C., Kimber, J., Davoli, M., 2009. Methadone maintenance therapy versus no opioid replacement therapy for opioid dependence. Cochrane Database Syst. Rev. 3 (CD002209).

McKenzie, M., Nunn, A., Zaller, N.D., Bazazi, A.R., Rich, J.D., 2009. Overcoming obstacles to implementing methadone maintenance therapy for prisoners: implications for policy and practice. J. Opioid Manag. 5, 219-227.

McKenzie, M., Zaller, N., Dickman, S.L., et al., 2012. A randomized trial of methadone initiation prior to release from incarceration. Subst. Abuse 33, 19-29.

McLellan, A.T., Luborsky, L., Woody, G.E., O’Brien, C.P., 1980. An improved diagnostic evaluation instrument for substance use patients. J. Nerv. Mental Dis. 168, 26-33.

Merrell, E.L.C., Kariminia, A., Binswanger, I.A., Hobbs, M.S., Farrell, M., Marsden, J., Hutchinson, S.J., Bird, S.M., 2010. Meta-analysis of drug-related deaths soon after release from prison. Addiction 105, 1545-1554.

Milloy, M.J., Buxton, J., Wood, E., Li, K., Julio, S.G., Kerr, T., 2009. Elevated HIV risk behaviour among recently incarcerated injection drug users in a Canadian setting: a longitudinal analysis. BMC Public Health 9, 156. 
Mumola, C.J., Karberg, J.C., 2006. Bureau of Justice Statistics Special Report: Drug Use and Dependence, State and Federal Prisoners, 2004. U.S. Department of Justice, Office of Justice Programs(https://www.bjs.gov/content/pub/pdf/dudsfp04.pdf. Accessed 05, November 2017)

Nunn, A., Zaller, N., Dickman, S., Trimbur, C., Nijhawan, A., Rich, J.D., 2009. Methadone and buprenorphine prescribing and referral practices in US prison systems: results from a nationwide survey. Drug Alcohol Depend. 105, 83-88.

Rhode Island Governor's Overdose Prevention and Intervention Task Force, 2015. Rhode Island's Strategic Plan on Addiction and Overdose: Four Strategies to Alter the Course of an Epidemic. Rhode Island Department of Health.

Rich, J.D., Chandler, R., Williams, B.A., et al., 2014. How health care reform can transform the health of criminal justice-involved individuals. Health Aff. 33, 462-467.

Rich, J.D., McKenzie, M., Larney, S., et al., 2015. Methadone continuation versus forced withdrawal on incarceration in a combined US prison and jail: a randomised, open- label trial. Lancet 386 (9991), 350-359.

Vestal, C., 2016. At Rikers Island, a Legacy of Medication-Assisted Opioid Treatment Stateline. The Pew Charitable Trusts.

Wakeman, S., 2017. Medications for addiction treatment: changing language to improve care. J. Addict. Med. 11, 1-2.

Wei, L.J., Lachin, John M., 1988. Properties of the urn randomization in clinical trials. Control. Clin. Trials 9, 345-364.

Zaller, N.D., Bazazi, A.R., Velazquez, L., Rich, J.D., 2009. Attitudes toward methadone among out-of-treatment minority injection drug users: implications for health disparities. Int. J. Environ. Res. Public Health 6, 787-797.

Zaller, N.D., McKenzie, M., Friedmann, P.D., Green, T.C., McGowan, S., Rich, J.D., 2013 Initiation of buprenorphine during incarceration and retention in treatment upon release. J. Subst. Abuse Treat. 45, 222-226. 